\title{
Evaluating Design Goodness Using Cluster Fuzzy Inference Algorithm
}

\author{
T. Freiheit \\ S. S. Park \\ simon.park@ucalgary.ca \\ C. N. Regier \\ tfreihei@ucalgary.ca \\ Department of Mechanical and Manufacturing Engineering \\ University of Calgary \\ Calgary, Alberta, Canada
}

\begin{abstract}
Fast changing global markets demand that manufacturers quickly develop products that are simultaneously costeffective and meet stakeholder needs. To survive in the hyper competitive environment of the information society, innovative product design is essential. However, it can be difficult for designers to identify whether the design is a "good" design before a product is manufactured and marketed. This paper develops a model to quantify the importance of good design characteristics. Through the use of cluster-fuzzy inference algorithm, quantified design "goodness" weights are generated based on surveys of rank ordered "goodness" characteristics. The cluster-fuzzy weights are compared with weights obtained from statistical analysis and found to have similar trends, but provides better insight to the relationship with rest of the characteristics. A cluster-fuzzy approach is an effective tool to determine important design parameters in the early stages of design.
\end{abstract}

\section{Introduction}

Global markets demand quick product development that is simultaneously cost-effective and meets stakeholder needs. With the advent of the information society, consumers now have greater access to a vast amount of information through the internet. Unlike in the past, where companies pushed out products that they hoped consumers would purchase, today's consumers are involved more with product development through various review forums. To survive in the hyper competitive environment of today's "knowledge based society," good product design is critical.

To be successful, designers wish to create designs that are unambiguously good. However, what does "good design" really mean? How do good designs contribute to success of companies? In order for products to be successful, what key ingredients are required? What factors affect the success of designed products? These are common questions that designers

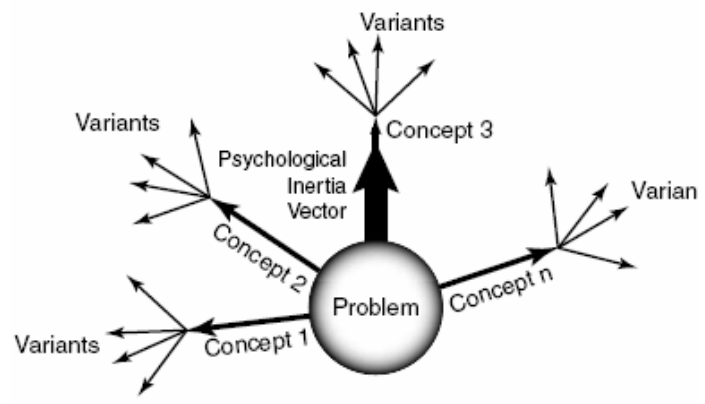

Figure 1. Trial and error design [1] ask. However, one obstacle in the realm of design is lack of meaningful measures for good design. Unless a good design can be quantitatively identified, the design can become a time-consuming iterative trial and error exercise, as shown in Figure 1. For example, Thomas Edison performed more than 6000 experiments to find a satisfactory light bulb filament material [2]. However, today's environment of severe competition does not permit an extensive trial and error process to develop good designs.

The objective of this work is to develop a model to quantify the importance of good design characteristics in a meaningful, repeatable way. While there are many benefits to quantifying the importance of each characteristic, the main benefit of this analysis is that different designers and industries have different opinions about which good design characteristic is most important, and a model must be flexible to this variation. Further, a quantified rating of different good design characteristics enables large volumes of design information to be focused, and makes decisions easier and easier to understand.

By setting a clear path from engineering decisions, to the design parameters, to the functional requirements of the design, and finally to the attributes of a good design, it not only becomes clear how specific engineering changes affect the overall goodness of design, but it also indicates how to achieve a desired increase in design quality from specific engineering decisions.

The outcome of this work is intended to be applied to a fuzzy logic model to provide guidance to designers when developing products. Fuzzy logic analysis uses a rule-based algorithm to determine whether a design is good, average or bad. Figure 2 depicts the 
fuzzy analysis process where a qualitative assessment of design characteristics is input, generating a qualitative assessment of the overall goodness of a particular design. In between the input and output is the inference engine, which consists of membership functions, fuzzy rule base, aggregation (or fuzzification) and defuzzification (converting fuzzy values into crisp values) processes. In providing this qualitative assessment, the fuzzy logic model requires the designer to supply a weight for good design characteristic, for which they have supplied their preferences through a rank ordering.

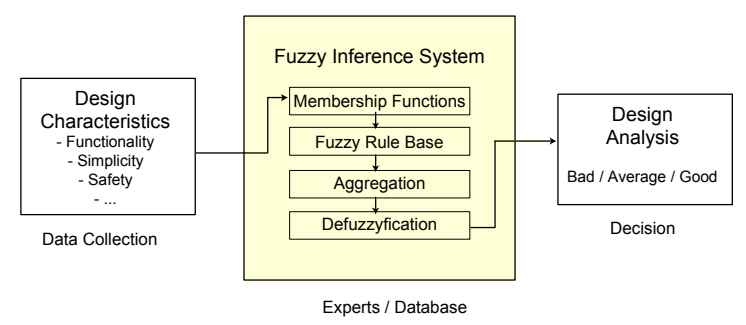

Figure 2. Fuzzy inference engine [3]

In this work, the cluster Adaptive Neuro-Fuzzy Inference System (ANFIS) algorithm is employed to effectively quantify "goodness" weights of the design parameters based on survey results. Unlike the traditional fuzzy logic where opinions of experts are required to form rule basis, the cluster fuzzy inference identifies the rules based on inputs and outputs [4]. The advantage of ANFIS analysis is that it is robust and simplifies knowledge acquisition and representation.

This paper is organized in the following manner: Section 2 surveys similar works done by other researchers; 13 characteristics of the good design attributes are listed in Section 3; Section 4 describes the processing of the surveyed results using the clusterfuzzy method; in Section 5, the determination of the cluster-fuzzy weight is presented based on the 13 goodness characteristics and examines necessary number of rules; and a summary is presented in the last section.

\section{Background}

Does a good design translate to business success? Roy [5] correlated good design to business success by surveying randomly selected firms vs. design leaders. $\mathrm{He}$ showed that firms that are design leaders have a return on capital that is $58 \%$ vs. $27 \%$ and profit margin is $14 \%$ vs. $1 \%$ to the randomly selected firms. This work clearly indicates that good design significantly affects business success, in addition to factors such as capability in the continuous development of products, adaptability to changing markets, and efficient manufacturing and selling.

Some publications have addressed characteristics of good design. Lidwell et al. [6] formulated 100 design guidelines that would benefit designers. Their design guidelines lack detail; however, they provide a comprehensive list of examples from wide ranges of designs. Seliger [2] reviewed innovative product developments, and determined that engineering science and design are interrelated, with social science deciding on goals. He comprehensively reviewed various design methodologies including TRIZ, the design processes of Pahl and Beitz, Axiomatic design, Design for X, Modular Design, Concurrent, and Cooperative Design.

Walsh [7] surveyed several companies involved with the design and manufacture of plastic products and found that among the firms with reputations for good design, many common characteristics were considered in the evaluation of their designs. These characteristics include appearance, suitability for use, efficiency in production and material use, marketing potential and profit potential, safety, durability, value for money, and a well planned design process.

Alexander et al. [8] conducted a review of literature related to good design practice for the medical field, and discuss methods such as Design for Manufacture, Design for Assembly, Design for Reliability, and Design for Usability. Each method is focused on designing a product to maximize a specific characteristic. It is important to note, however, that all of these characteristics may be important in creating a good design. Gardiner et al. [9] associate good designs with the amount of customer input.

Suh [10] devised an Axiomatic Design methodology based on independence and information axioms. According to Suh, good designs are defined as products with design parameters that independently satisfy functional requirements and minimize information content, also know as simplicity of designs. The simplicity of design has been stressed since the $14^{\text {th }}$ century logician Ockham's [6] stated that unnecessary elements decrease design efficiency and increase the probability of unanticipated challenges. Therefore, often simple designs represent robust designs which increase the signal to noise ratio. Norman [11] reflects on design as a cognitive scientist, where Visceral (beauty); Behavioral (usability); and Reflective (Pride of ownership) are important components of good design.

While these and other papers can assist in building a list of the characteristics of a good design, none of the above authors has attempted to assemble a complete list of these characteristics, nor has any of them tried to quantify the relative importance of each char- 
acteristic. Without a comprehensive, quantified list, it is impossible to quantify the overall goodness of designs adequately.

\section{Characteristics of Good Design}

In order to successfully market new products, the seller must have a clear target audience. According to Rogers [12], the most innovation have an S-shaped rate of adoption, as shown in Figure 3. Ryan and Gross [13] classified the target audience as a way of looking at how new products move through general population into categories as innovators, main stream, late adopters, and laggards. Figure 3 depicts the life cycle of products which relate the target audience and marketing effort.

No matter where a product is in its life cycle, marketing, business and technical factors must be associated with characteristics of a good product design. A relatively complete list of good design characteristics was assembled by reviewing the literature [3]. The following 13 factors, in no particular order, were identified as characteristics of a good design.

1. Marketing and Profit Potential

2. Safety During Use

3. Simplicity of Design

4. Simplicity of Use

5. Independent Functional Requirements

6. Uses Tried and True Technology

7. Durability

8. Robust to Variations in Operating Environment
9. Functionality - Does it do what it claims it will

10. Ease of Manufacturing

11. Aesthetics

12. Value for the Money or Expenditure

13. Well Planned \& Thought Out Creation Process.

The biggest difficulty in attempting to quantify the importance of each characteristic is individual differences in judgment or opinion about which is most important. For example, while one person may think that marketing potential is the most important characteristic for defining a design as good, another may be of the opinion that usability is more important. To address this issue, a survey was conducted to determine

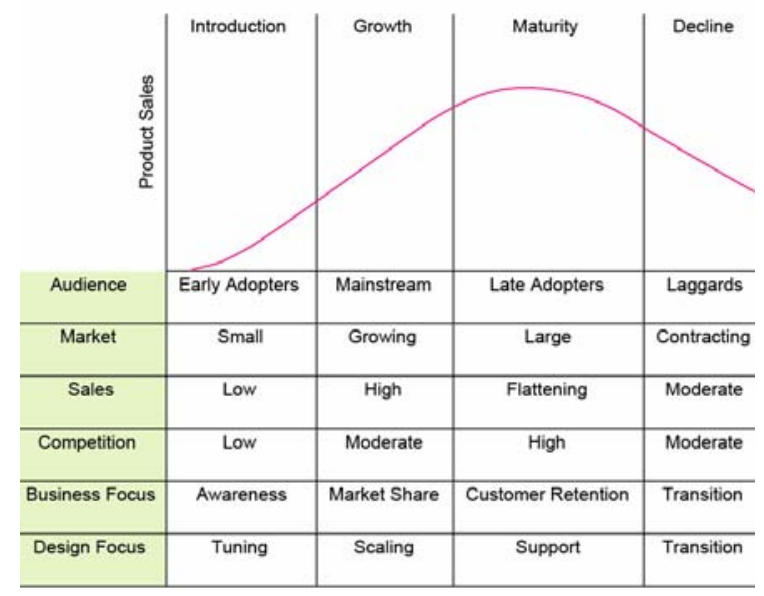

Figure 3. Life cycle of product: target audience and market [6]

\section{Table 1. Survey results - number of respondents for a given rank and characteristic [3]}

\begin{tabular}{|c|c|c|c|c|c|c|c|c|c|c|c|c|c|c|c|}
\hline \multirow[b]{2}{*}{ Good Design Characteristic } & \multicolumn{13}{|c|}{ Rank } & \multirow[b]{2}{*}{$\begin{array}{l}\text { Mean } \\
\text { Rank }\end{array}$} & \multirow[b]{2}{*}{$\begin{array}{l}\text { Std } \\
\text { Dev }\end{array}$} \\
\hline & 1 & 2 & 3 & 4 & 5 & 6 & 7 & 8 & 9 & 10 & 11 & 12 & 13 & & \\
\hline Marketing, or profit potential & 4 & 5 & 9 & 10 & 4 & 8 & 4 & 5 & 5 & 8 & 7 & 4 & 10 & 7.193 & 3.814 \\
\hline Safety during use & 17 & 10 & 7 & 9 & 6 & 8 & 8 & 6 & 5 & 3 & 3 & 1 & 0 & 4.771 & 3.125 \\
\hline Simplicity of design & 3 & 10 & 11 & 15 & 5 & 5 & 8 & 7 & 5 & 9 & 4 & 1 & 0 & 5.711 & 3.030 \\
\hline Simplicity of use & 2 & 18 & 15 & 11 & 13 & 7 & 7 & 2 & 2 & 2 & 2 & 2 & 0 & 4.614 & 2.608 \\
\hline $\begin{array}{l}\text { Independent functional re- } \\
\text { quirements }\end{array}$ & 0 & 1 & 3 & 3 & 2 & 5 & 3 & 7 & 13 & 7 & 15 & 13 & 11 & 9.530 & 2.847 \\
\hline Uses tried and true knowledge & 0 & 0 & 0 & 0 & 1 & 0 & 1 & 5 & 5 & 7 & 7 & 21 & 36 & 11.614 & 1.773 \\
\hline Durability & 0 & 2 & 9 & 8 & 13 & 10 & 10 & 11 & 8 & 6 & 3 & 2 & 1 & 6.602 & 2.580 \\
\hline $\begin{array}{l}\text { Robustness to variations in op } \\
\text { environment }\end{array}$ & 2 & 11 & 6 & 6 & 12 & 7 & 7 & 9 & 7 & 5 & 6 & 5 & 0 & 6.361 & 3.165 \\
\hline $\begin{array}{l}\text { Functionality - does it do what } \\
\text { it was intended to do }\end{array}$ & 47 & 18 & 5 & 6 & 2 & 3 & 1 & 1 & 0 & 0 & 0 & 0 & 0 & 1.988 & 1.573 \\
\hline Ease of manufacturing & 0 & 1 & 4 & 6 & 8 & 13 & 10 & 10 & 10 & 8 & 3 & 8 & 2 & 7.602 & 2.705 \\
\hline $\begin{array}{l}\text { Appearance is appealing to } \\
\text { people }\end{array}$ & 0 & 2 & 4 & 2 & 5 & 1 & 14 & 3 & 6 & 9 & 17 & 10 & 10 & 9.133 & 3.055 \\
\hline $\begin{array}{l}\text { Value for the money or expen- } \\
\text { diture }\end{array}$ & 2 & 2 & 6 & 3 & 5 & 10 & 8 & 12 & 9 & 10 & 8 & 7 & 1 & 7.699 & 2.946 \\
\hline $\begin{array}{l}\text { Well planned and thought out } \\
\text { creation process }\end{array}$ & 6 & 3 & 4 & 4 & 7 & 6 & 2 & 5 & 8 & 9 & 8 & 9 & 12 & 8.181 & 3.845 \\
\hline
\end{tabular}


the relative value people hold for these characteristics of good design, by having them rank order these 13 characteristics. A summary of how each good design characteristic is ranked is presented in Table 1.

The group chosen to take the survey was a fourth year mechanical and manufacturing engineering design class near the beginning of an eight month capstone design course. Benefits of choosing this group are that they are knowledgeable enough about design to contribute meaningful information, yet inexperienced enough with design so as not to have preconceived notions about answers. Thus the survey responses represent those of an educated consumer, thinking about the use of the product but also thinking about the design of the product. Disadvantages to choosing this group are that the individuals are all involved in a common career, which may somewhat bias the results.

A statistical analysis of the relative importance of each characteristic in this surveyed opinion was then used to quantify the value of each characteristic in determining whether a design is good. MultiDimensional Scaling (MDS) was used to distill the 83 respondents' characteristic rankings into a single rank ordering, reflecting the relative importance of each characteristic [3]. Table 2 lists the MDS adjusted rank.

\section{Cluster-Fuzzy Analysis}

As illustrated by Table 1 , the relative ranking of these 13 good design characteristics finds opinion varies as to which characteristic is most important, and how the characteristics should be rank ordered. While Multi-Dimensional Scaling (MDS) provides a good

Table 2. Survey ranking adjusted using multi-dimensional scaling [3]

\begin{tabular}{|l|c|}
\hline \multicolumn{1}{|c|}{ Characteristic } & Adjusted Rank \\
\hline $\begin{array}{l}\text { Functionality - does it do what it was } \\
\text { intended to do }\end{array}$ & 1 \\
\hline Simplicity of use & 3.7 \\
\hline Safety during use & 3.8 \\
\hline Simplicity of design & 4.9 \\
\hline Marketing, or profit potential & 6.1 \\
\hline $\begin{array}{l}\text { Robustness to variations in operating } \\
\text { environment }\end{array}$ & 6.1 \\
\hline Durability & 6.2 \\
\hline Ease of manufacturing & 8.5 \\
\hline Value for the money or expenditure & 8.5 \\
\hline $\begin{array}{l}\text { Well planned and thought out crea- } \\
\text { tion process }\end{array}$ & 10.0 \\
\hline Appearance is appealing to people & 10.4 \\
\hline Independent functional requirements & 11.0 \\
\hline Uses tried and true knowledge & 13.0 \\
\hline
\end{tabular}

overall ordering of the characteristics, just as the survey takers opinions varied, different designers or industries may value each characteristic differently. Thus it is useful to provide a means to calculate the characteristic weights for different characteristic rankings, yet still maintain a degree of the importance underlying each characteristic that is evident in Table 1.

An Adaptive Neuro-Fuzzy Inference System (ANFIS) model was selected to provide a characteristic weight with any arbitrary ranking order of the good design characteristics. This translation is accomplished through a set of fuzzy rules that transforms the characteristic ranking to weights. An example of the form of these rules is given in Figure 4 showing a 'four rule' system.

ANFIS uses a supervised learning algorithm to determine a nonlinear model of input and output function. There are several choices associated with ANFIS networks. One of the methods is subtractive clustering technique [14]. The subtractive clustering algorithm is a fast one-pass algorithm for estimating clusters based on the size of cluster centers by defining a radius.

In this study, Matlab's cluster-fuzzy 'GENFIS2' function [15] was utilized to generate a fuzzy inference system. One of the benefits of the clustering method is it reduces the redundancy of data and determine the rules and membership functions. The size of the cluster centers determines the number of rules associated with the fuzzy inference system (Figure 5). One of the drawbacks of the method is that the weighting functions may not always be always positive. In this analysis, the data from 80 students was used for the training and the additional 3 students' response were used to check the model.

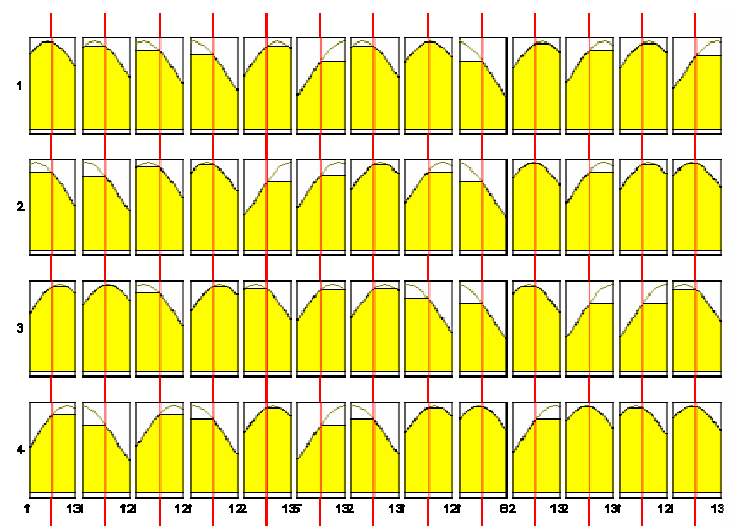

Figure 4. Example of fuzzy rule set (4 rules) 


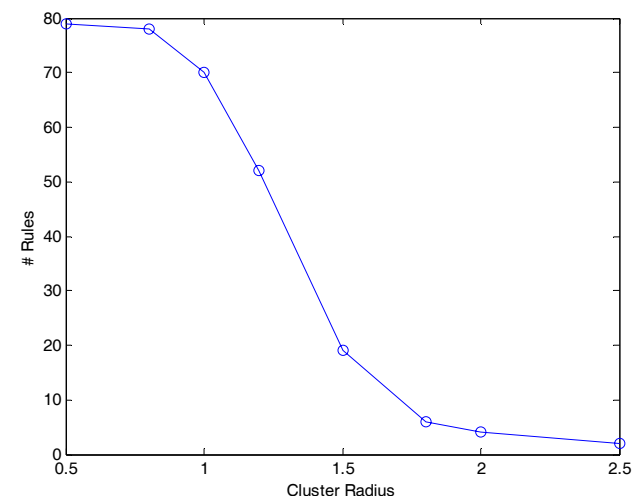

Figure 5. The relationship between the cluster size (radius) and the number of rules

In order to create this cluster-fuzzy model, it is necessary to translate these relative ranking into characteristic weights. However, relative ranking does not give a global weight of the perceived value for characteristic of a given design. A global weight can be identified by linking these ranked characteristic to the proportion of responses to unprompted, open ended survey questions classified as that characteristic [3]. The following characteristic weight to rank relationship was determined:
Char. Wt. $=-0.0825 \ln ($ Char. Rank $)+0.2201$

which provides a sum of approximately one for the thirteen characteristics.

Using this relationship, 80 survey responses which rank the 13 good design characteristics were used as input, and their associated characteristic weights were used as their 13 output to determine the cluster-fuzzy inference algorithm. This cluster-fuzzy algorithm gives a set of rules which will provide a characteristic weight for any ranking order of the characteristics. However, how much weight any ranked characteristic has is determined by the number of rules in the inference engine, which in turn influences the weighting error. Using the principle of Ockham's Razor [6], the minimum number of rules necessary to achieve an acceptable amount of error must be determined.

\section{Cluster-Fuzzy Characteristic Weight Determination}

For the purpose of this study, two factors are considered important in determining the acceptable level of error. First is how well the cluster-fuzzy characteristic weight matches the weight determined by multidimensional scaling of the ranking vectors' dataset. The second is that any arbitrary characteristic ranking

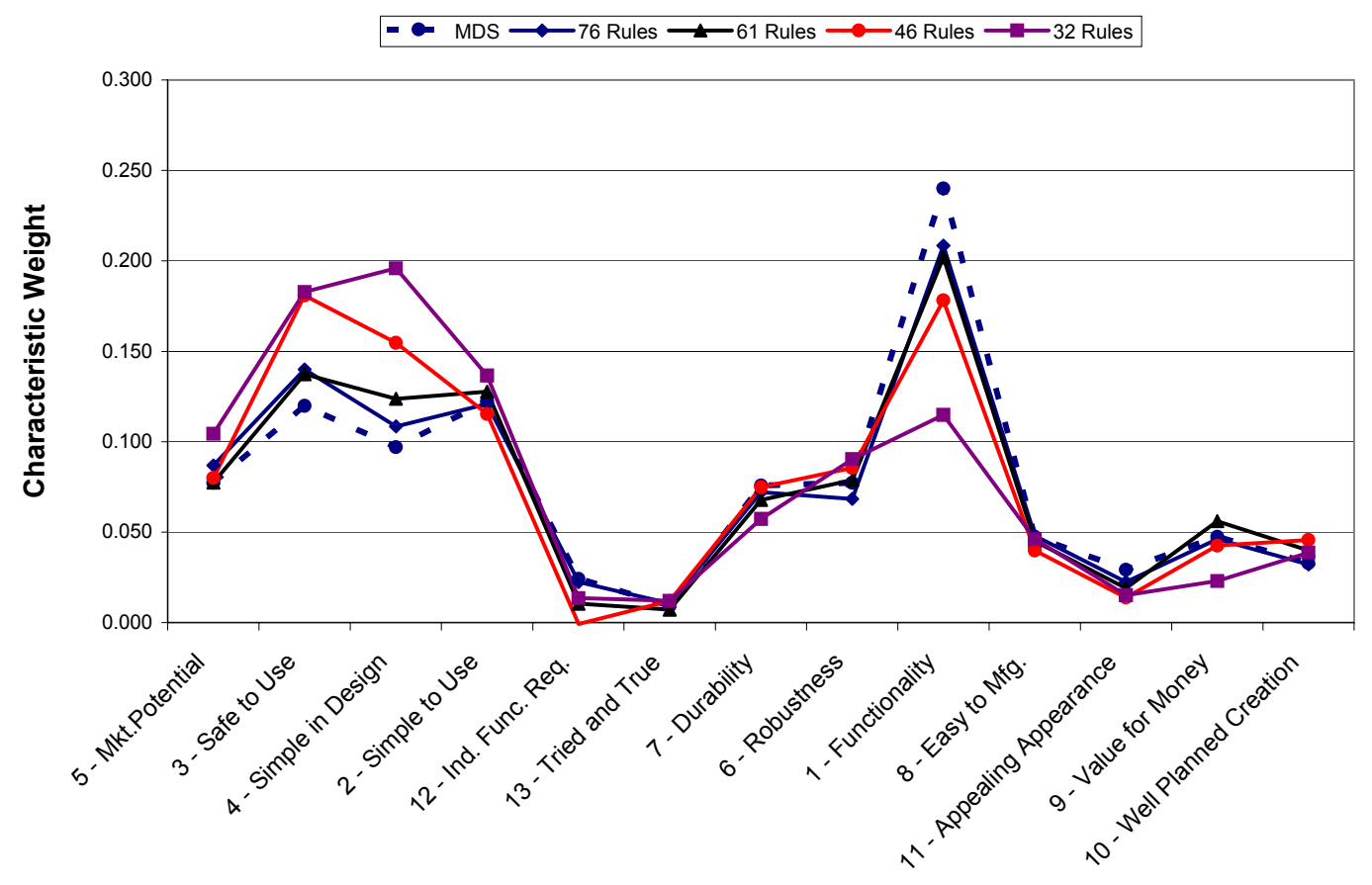

Good Design Characteristic

Figure 6. Number of Cluster-fuzzy Rules to Characteristic Weight Comparison 
should yield positive weights.

In the former factor, matching is deemed acceptable if a) the rule set provides weights similar to the MDS weights when tested with the MDS ranking, and b) when presented with an arbitrary ranking vector, the weight of the first ranked characteristic generally follows the MDS weighting curve. Requirement b) will be tested by generating multiple, random rankings of the characteristics, while fixing each characteristic in turn as first rank, and then comparing the mean weight determined for each characteristic to its MDS weight.

The requirement for positive weights for any arbitrary characteristic ranking set is necessary because by definition all characteristics are parameters contributing positively to a good design. This will be tested by examining the statistical distribution of weights obtained from random ranking, fixing each characteristic in turn as first rank.

Figure 6 compares the characteristic weight derived from different size cluster-fuzzy rule sets to the MDS weight when the thirteen parameters are ranked as shown. As is expected, as the number of rules is increased, the better the weights match the MDS weights. Figure 7 plots against the number of rules the mean square error of all characteristics and the percentage of negative characteristic weights when the cluster-fuzzy rule set is subjected to 160 random ranking's of the characteristics.
As can be seen in Figure 7, at approximately 60 rules, the level of deviation error from the MDS weights levels off, and the proportion of negative weights approaches zero. This plot also shows that at approximately 20 rules, the level of deviation error is on the order of that at 60 rules, but the number of negative weights is still high, making 20 rules unacceptable. Refining the analysis within the vicinity of 60 rules, it was determined that a minimum of 58 rules provides acceptable error.

With the 61 cluster-fuzzy rule set, characteristic weights were determined in thirteen runs where each characteristic was fixed as first rank and the other 12 characteristics were randomly ordered. Each run was for a minimum of 1000 random rank orderings, totaling over 13000 runs. This test determines if the weight of the first ranked characteristic generally follows the MDS weighting curve when presented with an arbitrary ranking vector, as well as examines if any cluster-fuzzy characteristic weight is negative.

Figure 8 plots the mean weight determined for each characteristic when that characteristic was ranked first, as well as two standard deviations of variation about its mean. For comparison, a bar chart of the MDS weights for the 13 characteristics ranked as shown is superimposed on the plot. This figure shows that the mean cluster-fuzzy weight generally matches the shape of the MDS weights, but as expected, are all

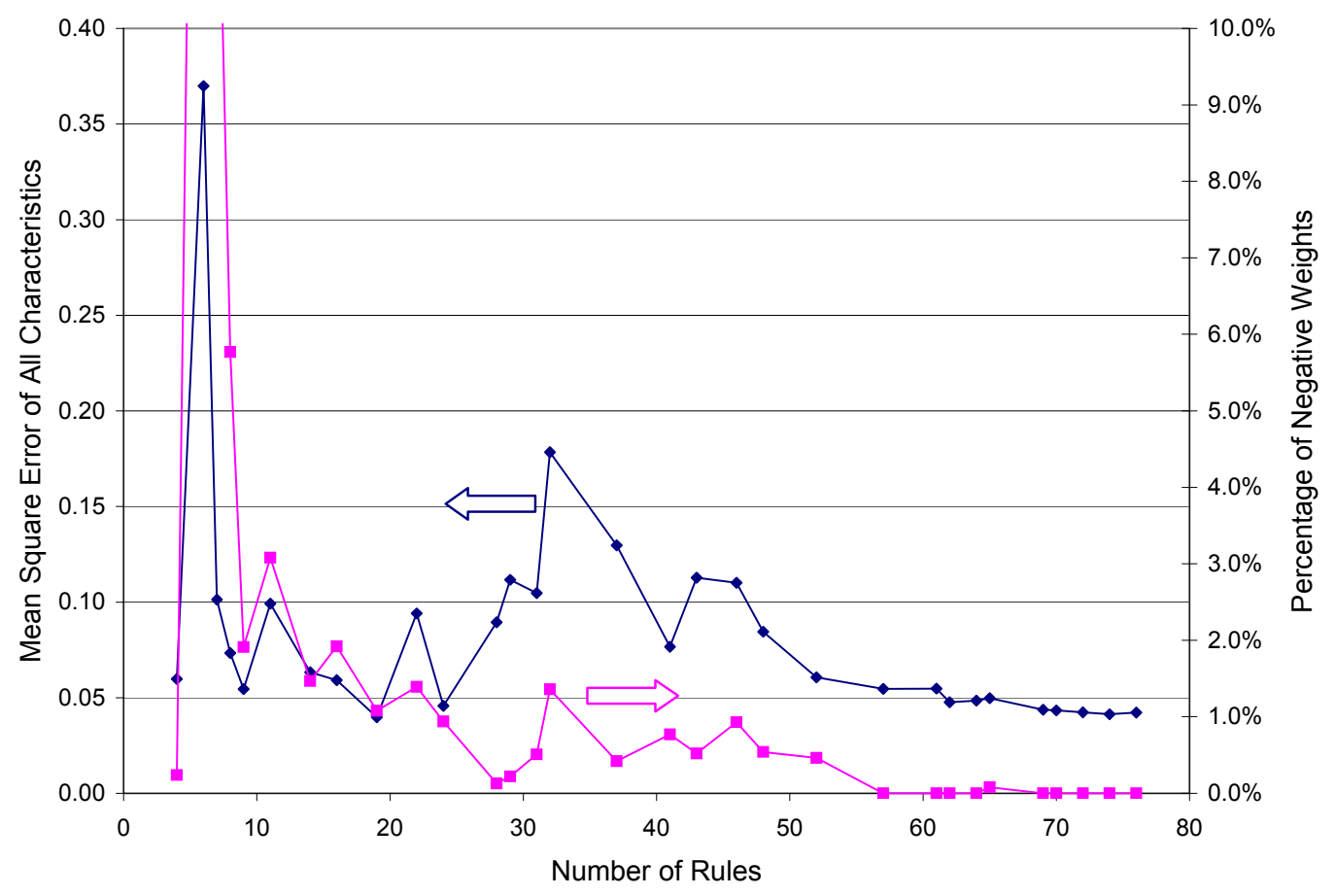

Figure 7. Error from cluster-fuzzy rule set size 


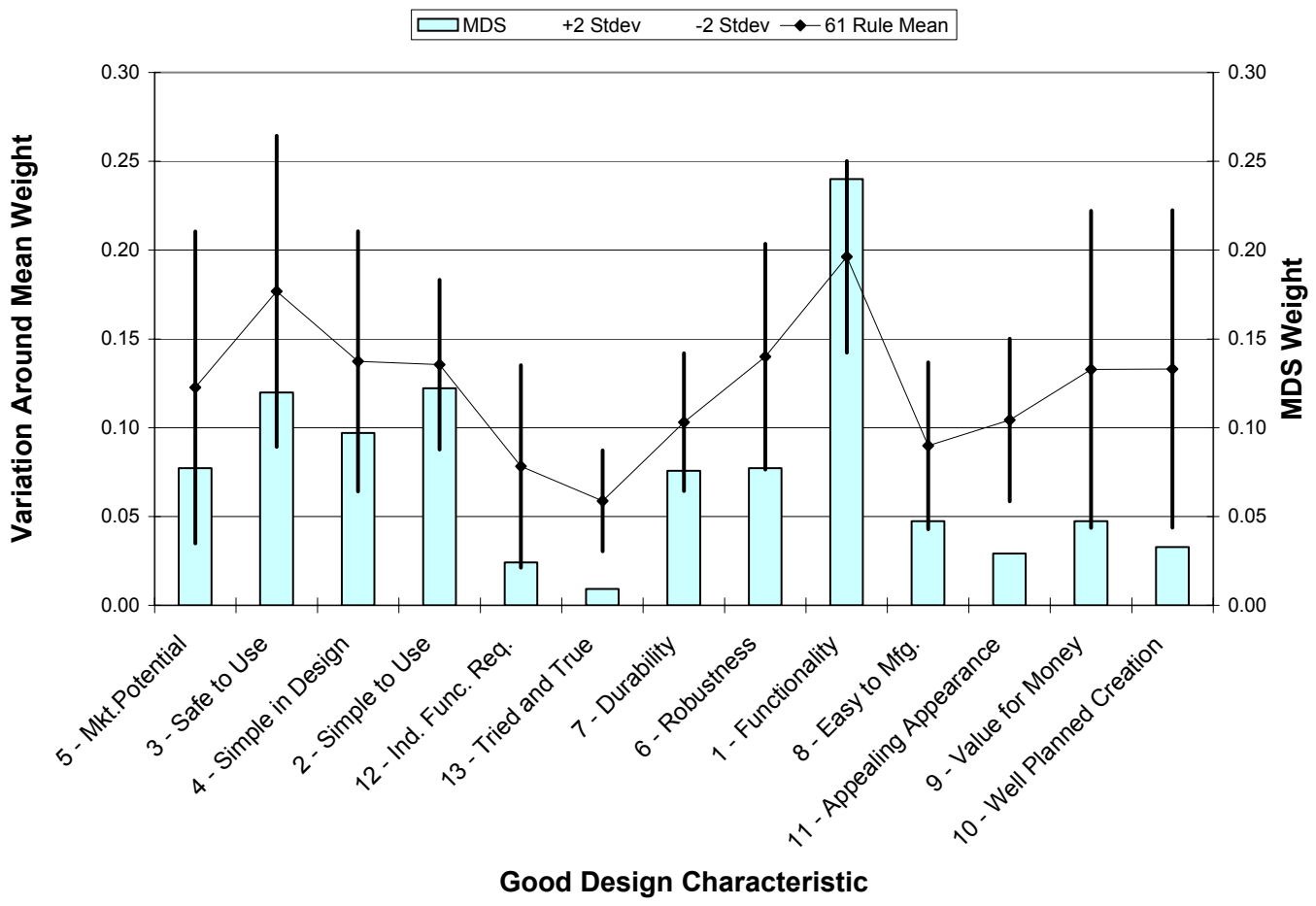

Figure 8. Variation around mean of first ranked characteristic (61 Rules)

higher except functionality. Intuitively, higher weights are expected because if a characteristic is rank ordered first, it should have a greater weight than the MDS, which ranked functionality first. 'Functionality' here is also be expected to have on average a lower weight than MDS, as this characteristic was most under weighted in the cluster-fuzzy model (Figure 6).

This random ranking test also determined that $0.1 \%-4.7 \%$ of the runs had one or more negative weights for its characteristics, with 'functionality' and 'a well planned creation process' ranked first having the most negative weights, and 'value for money' and 'appealing appearance' ranked first having the least negative weights. Examining when 'functionality' was ranked first, it was found that random rankings that produced negative weights generally occur in the condition where both low MDS rankings were ranked high, and high MDS rankings were ranked low, but is most sensitive to high ranking MDS ranked low. Since the survey indicated there is a trend in characteristic ranking as exhibited by the MDS ranking, this level of error is deemed acceptable.

Table 3 presents the results of 61 rule model with the three student's rank ordering reserved for that purpose. As can be seen, it generally follows the trend of the MDS data and does increase the weight of charac-

Table 3. Cluster-fuzzy output of student test rankings (61 rules)

\begin{tabular}{|c|c|c|c|c|c|c|}
\cline { 2 - 7 } \multicolumn{1}{c|}{} & \multicolumn{2}{c|}{ Student 1 } & \multicolumn{2}{c|}{ Student 2 } & \multicolumn{2}{c|}{ Student 3 } \\
\hline Char. & Rank & Wt. & Rank & Wt. & Rank & Wt. \\
\hline $\mathbf{1}$ & 7 & 0.071 & 4 & 0.078 & 6 & 0.103 \\
\hline $\mathbf{2}$ & 6 & 0.092 & 5 & 0.129 & 1 & 0.119 \\
\hline $\mathbf{3}$ & 5 & 0.094 & 3 & 0.136 & 4 & 0.064 \\
\hline $\mathbf{4}$ & 1 & 0.120 & 2 & 0.128 & 3 & 0.125 \\
\hline $\mathbf{5}$ & 9 & 0.034 & 6 & 0.056 & 13 & 0.005 \\
\hline $\mathbf{6}$ & 13 & 0.016 & 12 & 0.005 & 12 & 0.007 \\
\hline $\mathbf{7}$ & 11 & 0.038 & 8 & 0.060 & 10 & 0.035 \\
\hline $\mathbf{8}$ & 8 & 0.059 & 7 & 0.066 & 8 & 0.064 \\
\hline $\mathbf{9}$ & 4 & 0.191 & 1 & 0.206 & 2 & 0.111 \\
\hline $\mathbf{1 0}$ & 12 & 0.025 & 11 & 0.026 & 7 & 0.041 \\
\hline $\mathbf{1 1}$ & 10 & 0.029 & 10 & 0.025 & 9 & 0.035 \\
\hline $\mathbf{1 3}$ & 3 & 0.084 & 9 & 0.030 & 11 & 0.024 \\
\hline $\mathbf{1 3}$ & 2 & 0.110 & 13 & 0.009 & 5 & 0.117 \\
\hline
\end{tabular}


teristics that are ranked 1 , e.g. student 1 ranked characteristic 'well planned creation process' (no. 13) as second, giving a fuzzy-model weight of 0.110 , significantly higher than the MDS weight of 0.033. However, because the fuzzy-model weighting is influenced by the distribution of ranking given by the other 80 students, an overall comparison to the fuzzy-model output weights to those generated from Equation (1) (used as output for the training of the cluster-fuzzy algorithm) is not meaningful.

\section{Summary}

In this work, we have listed thirteen attributes associated with "goodness" of a design. The relative importance of these design characteristics was ranked by 83 undergraduate mechanical and manufacturing engineering students. As indicated by this data, the ranking of these characteristics varies depending on background of the respondent and at what point in the life cycle shown in Figure 3 the product they had in their mind when they filled out the survey. To account for these differences, a fuzzy-cluster method of determining the value, or weight, of the design characteristics has been developed.

With these weights, a designer can estimate the effectiveness of their design in each of these 13 areas using a weighted sum or fuzzy calculation as described by [3] to arrive at a number quantifying the goodness of their design. When combined with other existing design tools, it is possible to create an algorithm that will indicate how low-level engineering decisions or selections may influence the goodness of a design.

Future work will include generating additional survey data more comprehensively exploring attitudes about good design, analyzing additional case studies to test the goodness rating, and developing fuzzy rules that can more fully describe the relationship between good design characteristic weights and qualitative assessments of overall design goodness.

\section{References}

[1] Fey, V. R., Rivin, E. L., The Science Of Innovation, TRIZ Group, Michigan, 1997.

[2] Seliger, G., "Product Innovation - Industrial Approach", Annals of CIRP, 50 (2), 2001.

[3] Regier, C.N., Freiheit, T., Park, S.S., "A Fuzzy Tool to Determine the Quality of Design based on Survey Results", CIRP Design Conference, Kananaskis, 2006.

[4] Jang, J.S.R., Sun, C.T., Mizutani, E., Neuro-Fuzzy and Soft Computing, Prentice Hall, NJ., 1997.

[5] Roy, R., "Can the Benefits of Good Design Be Quantified”, Design Management Journal, 5 (2), 1994, pp. 9-17.
[6] Lidwell, W., Holden, K., Butler, J., Universal Principles of Design, Rockport, Massachusetts, 2003.

[7] Walsh, V., "Plastic Products: Successful Firms, Innovation, and Good Design”, Design Studies, 4 (1), 1983, pp. 312.

[8] Alexander, K. and Clarkson, P., "Good Design Practice for Medical Devices and Equipment, Part 1: A Review of Current Literature", Journal of Medical Engineering and Technology, 24 (1), 2000, pp. 5-13.

[9] Gardiner, P., Rothwell, R., "Tough Customers, Good Designs”, Design Studies, 6 (1), 1985, pp. 7-17.

[10] Suh, N., Principles of Design, Oxford University Press, 1990.

[11] Norman, D.A., The Design of Everyday Things, Basic Books, New York, 1988.

[12] Rogers, Everett M., Diffusion of Innovations, Fress Press, New York, 1995.

[13] Ryan, B., Gross, N. C., "The diffusion of hybrid seed corn in two Iowa communities", Rural Sociology, 8, 1943, pp. 15-24.

[14] Chiu, S., "Fuzzy Model Identification Based on Cluster Estimation," Journal of Intelligent \& Fuzzy Systems, 2 (3) 1994.

[15] Matlab ${ }^{\mathrm{TM}}$, Fuzzy Toolbox. 